\title{
A massive planet to the young disc star HD 81040^
}

\author{
A. Sozzetti ${ }^{1,2}$, S. Udry ${ }^{3}$, S. Zucker ${ }^{4,5}$, G. Torres $^{1}$, J. L. Beuzit ${ }^{6}$, D. W. Latham ${ }^{1}$,

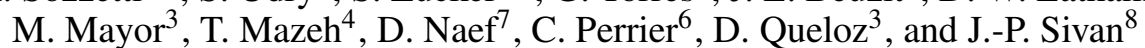 \\ ${ }^{1}$ Harvard-Smithsonian Center for Astrophysics, 60 Garden Street, Cambridge, MA 02138, USA \\ e-mail: asozzett@cfa.harvard.edu \\ 2 INAF - Osservatorio Astronomico di Torino, 10025 Pino Torinese, Italy \\ 3 Observatoire de Genève, 51 Ch. de Maillettes, 1290 Sauveny, Switzerland \\ ${ }^{4}$ School of Physics and Astronomy, Raymond and Beverly Sackler Faculty of Exact Sciences, Tel Aviv University, Tel Aviv 69978, Israel \\ 5 Department of Geophysics and Planetary Sciences, Beverly and Raymond Sackler Faculty of Exact Sciences, Tel Aviv University, \\ Tel Aviv 69678, Israel \\ ${ }^{6}$ Laboratoire d'Astrophysique de Grenoble, Université J. Fourier, BP 53, 38041 Grenoble, France \\ 7 European Southern Observatory, 3107 Alonso de Cordova, Casilla 19001, Santiago 19, Chile \\ 8 Observatoire de Haute-Provence, 04870 St-Michel l'Observatoire, France
}

Received 5 October 2005 / Accepted 23 November 2005

\section{ABSTRACT}

We report the discovery of a massive planetary companion orbiting the young disc star HD 81040. Based on five years of precise radial-velocity measurements with the HIRES and ELODIE spectrographs, we derive a spectroscopic orbit with a period $P=1001.0$ days and eccentricity $e=0.53$. The inferred minimum mass for the companion of $m_{2} \sin i=6.86 M_{\text {Jup }}$ places it in the high-mass tail of the extrasolar planet mass distribution. From the ELODIE spectra we derive a Lithium abundance of $\log \epsilon(\mathrm{Li})=1.90$, and from the HIRES spectra of the cores of the $\mathrm{Ca}$ II $\mathrm{H}$ and $\mathrm{K}$ lines we derive an activity index of $\left\langle\log R_{\mathrm{HK}}^{\prime}\right\rangle=-4.48$, suggesting an age of about 0.8 Gyr. The radial-velocity residuals exhibit a scatter significantly larger than the typical internal measurement precision of the instruments. We attribute this excess velocity jitter to activity on the surface of the moderately young host star. However, the amplitude of the jitter is much too small and the expected period of rotation is much too short to explain the observed orbital motion, which we conclude is due to a massive planetary companion.

Key words. stars: planetary systems - stars: individual: HD 81040 - stars: activity - stars: abundances - techniques: radial velocities techniques: spectroscopic

\section{Introduction}

Except for one experiment (Cochran et al. 2002), radialvelocity surveys for planets orbiting nearby, solar-type stars with a targeted precision of 1-5 $\mathrm{m} \mathrm{s}^{-1}$ (e.g., Mayor et al. 2004; Santos et al. 2004; Butler et al. 2004; Marcy et al. 2005a,b) have systematically attempted to exclude from their samples chromospherically active stars. This is because changes in the visibility of active regions and variations of the stellar absorption line profiles can possibly cause significant deterioration in the achievable radial-velocity precision. However, for about a dozen planet hosts significant activity levels have not prevented radial-velocity surveys from detecting orbital motion due to giant planets. In this paper we report radial-velocity

* Based on observations made at the Observatoire de HauteProvence (CNRS, France) and at the W. M. Keck Observatory, which is operated as a scientific partnership among the California Institute of Technology, the University of California and the National Aeronautics and Space Administration. The Observatory was made possible by the generous financial support from the W. M. Keck Foundation. measurements for the young disc star HD 81040 (HIP 46076, $\left.\mathrm{BD}+20^{\circ} 2314\right)$. These observations reveal the presence of a massive planet candidate orbiting the star, with a minimum mass of $6.86 M_{\text {Jup }}$ (where $M_{\text {Jup }}$ is the mass of Jupiter).

The variable velocity of HD 81040 was first detected by the G-Dwarf Planet Search (Latham 2000), a program designed to conduct a first reconnaissance for giant planets orbiting a sample of nearly 1000 nearby $\mathrm{G}$ dwarfs. This survey employed the HIRES spectrograph (Vogt et al. 1994) on the 10-m Keck 1 telescope at the W. M. Keck Observatory (Hawaii). Followup observations were carried out within the context of the ELODIE Planet Search Survey (Mayor \& Queloz 1995; Perrier et al. 2003), which uses the ELODIE fiber-fed echelle spectrograph (Baranne et al. 1996) on the 1.93-m telescope at the Observatoire de Haute-Provence (CNRS, France).

Radial velocities with ELODIE are obtained by crosscorrelating the observed spectra with a numerical template. The "simultaneous thorium-argon technique" with dual fibers (Baranne et al. 1996) allows for the monitoring and correction of instrumental drifts. The precision achieved with this 
instrument on bright, inactive stars is $\sim 6.5 \mathrm{~m} \mathrm{~s}^{-1}$ (Perrier et al. 2003). The HIRES instrumental profile and drifts are monitored using an iodine absorption cell (Marcy \& Butler 1992). In order to derive radial velocities, the data reduction procedure involves the modelling of the temporal and spatial variations of the instrumental profile of the spectrograph (Valenti et al. 1995), and is conceptually similar to that described by Butler et al. (1996). Internal errors, computed from the scatter of the velocities from the echelle orders containing $I_{2}$ lines, are typically $8-10 \mathrm{~m} \mathrm{~s}^{-1}$ for inactive, solar-type stars, for the relatively short exposures adopted for the G-dwarf Planet Search.

The collaboration between our two teams has so far resulted in three important discoveries: the determination of the spectroscopic orbit of the first extrasolar planet transiting the disc of its parent star (HD 209458b; Mazeh et al. 2000), the detection of HD $80606 \mathrm{~b}$, the planet with the largest eccentricity known to-date ( $e=0.927$, Naef et al. 2001a), and the identification of the first giant planet in a stellar triple system (HD 178911Bb, Zucker et al. 2002).

The Keck/HIRES observations of HD 81040 began in April 1999 and have a time baseline of $\sim 8.5$ months. The ELODIE follow-up observations span $\sim 3.3$ years, starting in February 2002. We present in Sect. 2 the radial-velocity data and the orbital solution derived utilizing the combined datasets. The stellar characteristics of HD 81040 are considered in Sect. 3. Finally, Sect. 4 is devoted to a summary and discussion of our findings.

\section{Radial-velocity data and orbital solution}

The 26 radial velocity measurements for HD 81040 are listed in Table 1, where the HIRES velocities have been shifted by $49.24 \mathrm{~km} \mathrm{~s}^{-1}$ to bring them close to the ELODIE system. A small residual velocity offset $\Delta R V_{\mathrm{H}-\mathrm{E}}=+0.057 \mathrm{~km} \mathrm{~s}^{-1}$ between the two systems was determined as a free parameter in the best-fit orbital solution presented in Table 2. We show in the upper and lower panel of Fig. 1 the radial-velocity measurements as a function of time and orbital phase, respectively. Post-fit velocity residuals are shown in the two sub-panels. Using the value for the primary mass of $M_{\star}=0.96 \pm 0.04 M_{\odot}$ obtained as described in Sect. 3, we derive a minimum mass for the companion $m_{2} \sin i=6.86 \pm 0.71 M_{\mathrm{Jup}}$. The radial-velocity variations measured in HD 81040 thus reveal the presence of a long-period $(P \simeq 2.7$ years $)$, eccentric $(e=0.53)$, massive planetary-mass object. Due to gaps in phase coverage and limited number and time baseline of the observations, further measurements will be required to improve on the determination of some of the orbital elements (such as the orbital period and the eccentricity).

Given the values of $m_{2} \sin i$ and $P$, translating into an astrometric signature $\alpha \approx 410 \mu$ as at the distance of HD 81040, we have investigated the Hipparcos Intermediate Astrometric Data (IAD) in an attempt to further constrain the companion mass (for a review of the method see for example Sozzetti 2005, and references therein). The star is flagged as single in the Hipparcos database. The best-fit astrometric solution with the period of the spectroscopic orbit gives a statistically insignificant semi-major axis of $2.0 \pm 1.0$ mas. We have then
Table 1. Radial velocity measurements of HD 81040. The HIRES (H) velocities are shifted to the ELODIE (E) zero point.

\begin{tabular}{lccc}
\hline \hline BJD - 2400 000 & $\begin{array}{c}\text { Radial velocity } \\
\left(\mathrm{km} \mathrm{s}^{-1}\right)\end{array}$ & $\begin{array}{c}\sigma_{\mathrm{RV}} \\
\left(\mathrm{km} \mathrm{s}^{-1}\right)\end{array}$ & Instrument \\
\hline 51291.36542 & 49.319 & 0.012 & $\mathrm{H}$ \\
51293.37376 & 49.312 & 0.014 & $\mathrm{H}$ \\
51545.63921 & 49.088 & 0.009 & $\mathrm{H}$ \\
52308.63310 & 49.399 & 0.012 & $\mathrm{E}$ \\
52356.41490 & 49.412 & 0.021 & $\mathrm{E}$ \\
52359.45970 & 49.363 & 0.012 & $\mathrm{E}$ \\
52360.42310 & 49.391 & 0.011 & $\mathrm{E}$ \\
52615.71400 & 49.119 & 0.011 & $\mathrm{E}$ \\
52649.66360 & 49.126 & 0.011 & $\mathrm{E}$ \\
52649.67630 & 49.129 & 0.013 & $\mathrm{E}$ \\
52719.40970 & 49.100 & 0.008 & $\mathrm{E}$ \\
52722.49520 & 49.118 & 0.012 & $\mathrm{E}$ \\
52993.63610 & 49.204 & 0.012 & $\mathrm{E}$ \\
53034.56760 & 49.293 & 0.015 & $\mathrm{E}$ \\
53094.36570 & 49.308 & 0.010 & $\mathrm{E}$ \\
53101.39950 & 49.285 & 0.010 & $\mathrm{E}$ \\
53359.69070 & 49.389 & 0.016 & $\mathrm{E}$ \\
53361.66000 & 49.412 & 0.021 & $\mathrm{E}$ \\
53421.52680 & 49.459 & 0.019 & $\mathrm{E}$ \\
53428.48940 & 49.462 & 0.026 & $\mathrm{E}$ \\
53461.36050 & 49.414 & 0.016 & $\mathrm{E}$ \\
53463.41260 & 49.458 & 0.020 & $\mathrm{E}$ \\
53464.39850 & 49.408 & 0.011 & $\mathrm{E}$ \\
53486.35380 & 49.359 & 0.011 & $\mathrm{E}$ \\
53491.32410 & 49.348 & 0.010 & $\mathrm{E}$ \\
53518.35250 & 49.234 & 0.013 & $\mathrm{E}$ \\
\hline
\end{tabular}

Table 2. Best-fit orbital solution for HD 81040, derived minimum companion mass, and properties of the post-fit residuals.

\begin{tabular}{lc}
\hline \hline Parameter & Value \\
\hline$P($ days $)$ & $1001.7 \pm 7.0$ \\
$T(\mathrm{BJD})$ & $2452504.0 \pm 12.0$ \\
$e$ & $0.526 \pm 0.042$ \\
$\gamma\left(\mathrm{km} \mathrm{s}^{-1}\right)$ & $+49.2535 \pm 0.0063$ \\
$\omega\left({ }^{\circ}\right)$ & $81.3 \pm 7.2$ \\
$K_{1}\left(\mathrm{~m} \mathrm{~s}^{-1}\right)$ & $168 \pm 9$ \\
$\Delta R V_{\mathrm{H}-\mathrm{E}}\left(\mathrm{km} \mathrm{s}^{-1}\right)$ & $+0.057 \pm 0.019$ \\
$a_{1} \sin i\left(\times 10^{6} \mathrm{~km}\right)$ & $1.96 \pm 0.19$ \\
$f_{1}(m)\left(\times 10^{-7} M_{\odot}\right)$ & $3.0 \pm 0.9$ \\
$m_{2} \sin i\left(M_{\mathrm{Jup}}\right)$ & $6.86 \pm 0.71$ \\
$a(\mathrm{AU})$ & 1.94 \\
$N$ & $23(\mathrm{E})+3(\mathrm{H})$ \\
$\left\langle\sigma_{\mathrm{RV}}\right\rangle\left(\mathrm{m} \mathrm{s}^{-1}\right)$ & 13.7 \\
$\sigma_{\mathrm{O}-\mathrm{C}}\left(\mathrm{m} \mathrm{s}^{-1}\right)$ & $26.0(\mathrm{E}: 25.0, \mathrm{H}: 6.0)$ \\
$\chi_{v}^{2}$ & 3.26 \\
$P r\left(\chi^{2}\right)$ & $2 \times 10^{-9}$ \\
\hline
\end{tabular}

used these values to derive an upper limit to the actual size of the astrometric orbit $\alpha$ with a $2.3 \sigma$ confidence, following the procedure described in Zucker \& Mazeh (2001). The resulting upper limit on the companion mass of $\sim 75 M_{\text {Jup }}$, at the $99 \%$ confidence level, clearly indicates its sub-stellar nature.

The scatter $\sigma_{\mathrm{O}-\mathrm{C}}$ of the post-fit residuals reported in Table 2 is uncomfortably large when compared to the average $\left\langle\sigma_{\mathrm{RV}}\right\rangle$ of the internal errors for both instruments. Inspection of the 

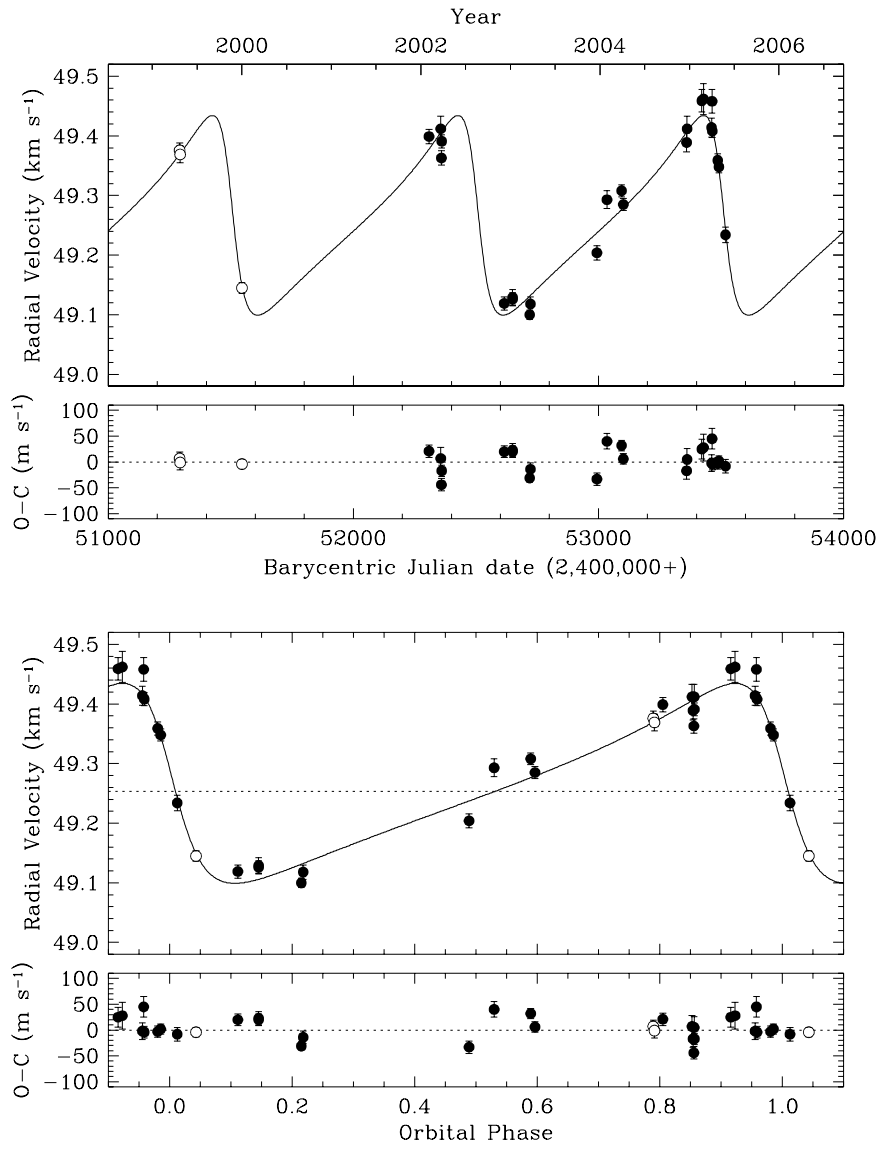

Fig. 1. Top: radial-velocity measurements as a function of time for HD 81040. Open circles identify Keck/HIRES velocities, while filled black circles correspond to the ELODIE dataset. Bottom: radial velocities as a function of orbital phase. The two sub-panels show the post-fit residuals in both cases.

velocity residuals (top and bottom sub-panels of Fig. 1) by means of a periodogram search revealed no additional periodicity within the time-span of the observations. No significant velocity trend is apparent in the post-fit residuals (a linear fit to the residuals time series had an insignificant slope of $\left.1.8 \pm 2.2 \mathrm{~m} \mathrm{~s}^{-1} \mathrm{yr}^{-1}\right)$. Thus, another companion on a longerperiod orbit superposing a second radial-velocity signal is an unlikely explanation (although additional measurements might clarify this issue). Another possibility to explain the excess jitter in the residuals is to invoke effects due to chromospheric activity of the parent star (e.g., Baliunas et al. 1995; Saar et al. 1998; Santos et al. 2000a). We discuss this possibility in the next section.

\section{Properties of the host star}

HD 81040 (HIP 46076, BD+20 2314) is a bright, nearby dwarf. Hipparcos astrometry places it at $32.56 \mathrm{pc}$ from the Sun. The quoted visual magnitude and color index from Hipparcos are $m_{V}=7.72$ and $B-V=0.68$, respectively. The corresponding absolute magnitude is $M_{V}=5.17$, consistent with a G2/G3 spectral type (Cox 2000), and this classification is supported by our effective temperature determination, $T_{\text {eff }}=5700 \mathrm{~K}$ (see Sect. 3.1). A G0 spectral type is reported in
Table 3. Observed and inferred stellar parameters for HD 81040. Spectral type, apparent magnitude, colour index, parallax, and proper motions are from Hipparcos (ESA 1997). The atmospheric parameters $T_{\text {eff }}, \log g, \xi_{t}$, and $[\mathrm{Fe} / \mathrm{H}]$, and the stellar mass and radius have been derived as described in Sect. 3.1. The values of $v \sin i,\left\langle\log R_{\mathrm{HK}}^{\prime}\right\rangle$, $\log \epsilon(\mathrm{Li})$, and age have been obtained as described in Sect. 3.2.

\begin{tabular}{lc}
\hline \hline Parameter & Value \\
\hline Sp. type & $\mathrm{G} 2 / \mathrm{G} 3$ \\
$m_{V}$ & 7.72 \\
$B-V$ & 0.68 \\
$\pi(\mathrm{mas})$ & $30.71 \pm 1.24$ \\
$d(\mathrm{pc})$ & $32.56 \pm 1.31$ \\
$\mu_{\alpha}\left(\mathrm{mas} \mathrm{yr}^{-1}\right)$ & $-151.35 \pm 1.08$ \\
$\mu_{\delta}\left(\mathrm{mas} \mathrm{yr}^{-1}\right)$ & $35.91 \pm 0.52$ \\
$M_{V}$ & 5.17 \\
$B . C$. & 0.10 \\
$(U, V, W)\left(\mathrm{km} \mathrm{s}^{-1}\right)$ & $(40.9,-1.5,25.1)$ \\
$T_{\text {eff }}(\mathrm{K})$ & $5700 \pm 50$ \\
$\log g\left(\mathrm{cgs}^{2}\right)$ & $4.5 \pm 0.1$ \\
$\left.\xi_{t}(\mathrm{~km} \mathrm{~s})^{-1}\right)$ & $0.95 \pm 0.05$ \\
{$[\mathrm{Fe} / \mathrm{H}]$} & $-0.16 \pm 0.06$ \\
$M_{\star}\left(M_{\odot}\right)$ & $0.96 \pm 0.04$ \\
$R_{\star}\left(R_{\odot}\right)$ & $0.86 \pm 0.04$ \\
$\left\langle\log R_{\mathrm{HK}}^{\prime}\right\rangle$ & -4.48 \\
$v \sin i\left(\mathrm{~km} \mathrm{~s}{ }^{-1}\right)$ & $2 \pm 1$ \\
$\log \epsilon(\mathrm{Li})$ & $1.90 / 1.91 \pm 0.07$ \\
$\langle t\rangle(\mathrm{Gyr})$ & $0.73 \pm 0.1$ \\
\hline
\end{tabular}

SIMBAD and several other sources, but the ultimate source for all these is the Henry Draper catalog. Using proper motions and parallax from Hipparcos and the $\gamma$ velocity obtained from the orbital solution described in Sect. 2 (in good agreement with the Nordström et al. (2004) reported value of $\left.48.9 \pm 0.2 \mathrm{~km} \mathrm{~s}^{-1}\right)$, the Galactic velocity vector for HD 81040 (corrected for the Local Standard of Rest following Mihalas \& Binney 1981) is then $(U, V, W)=(40.9,-1.5,25.1) \mathrm{km} \mathrm{s}^{-1}$. These values are summarized in Table 3.

\subsection{Abundance analysis}

We have utilized the Keck/HIRES high-signal-to-noise template spectrum of HD 81040 to carry out an LTE spectroscopic iron abundance analysis. In this study, we have followed the approach described in previous works (Sozzetti et al. 2004, 2006, and references therein). Our final iron list consisted of $28 \mathrm{Fe} \mathrm{I}$ and $6 \mathrm{Fe}$ II lines (taken from Sozzetti et al. 2006). The final atmospheric parameters, summarized in Table 3, are: $T_{\text {eff }}=5700 \pm 50 \mathrm{~K}, \log g=4.5 \pm 0.1(\mathrm{cgs}), \xi_{t}=0.95 \pm$ $0.05 \mathrm{~km} \mathrm{~s}^{-1}$, and $[\mathrm{Fe} / \mathrm{H}]=-0.16 \pm 0.06$. All these numbers are in very good agreement with those reported by Allende Prieto \& Lambert (1999) and Nordström et al. (2004).

The spectroscopically determined values of $T_{\text {eff }}$ and $[\mathrm{Fe} / \mathrm{H}]$, and the absolute luminosity estimate, were then used as input to the Yale stellar evolution models (Yi et al. 2003) to derive estimates of the stellar mass and radius for HD 81040, as well as their uncertainties. The results, also reported in Table 3 , are as follows: $M_{\star}=0.96 \pm 0.04 M_{\odot}, R_{\star}=0.86 \pm 0.04 R_{\odot}$. The predicted $\log g$ value is 4.52 , in excellent agreement with the spectroscopic estimate. 

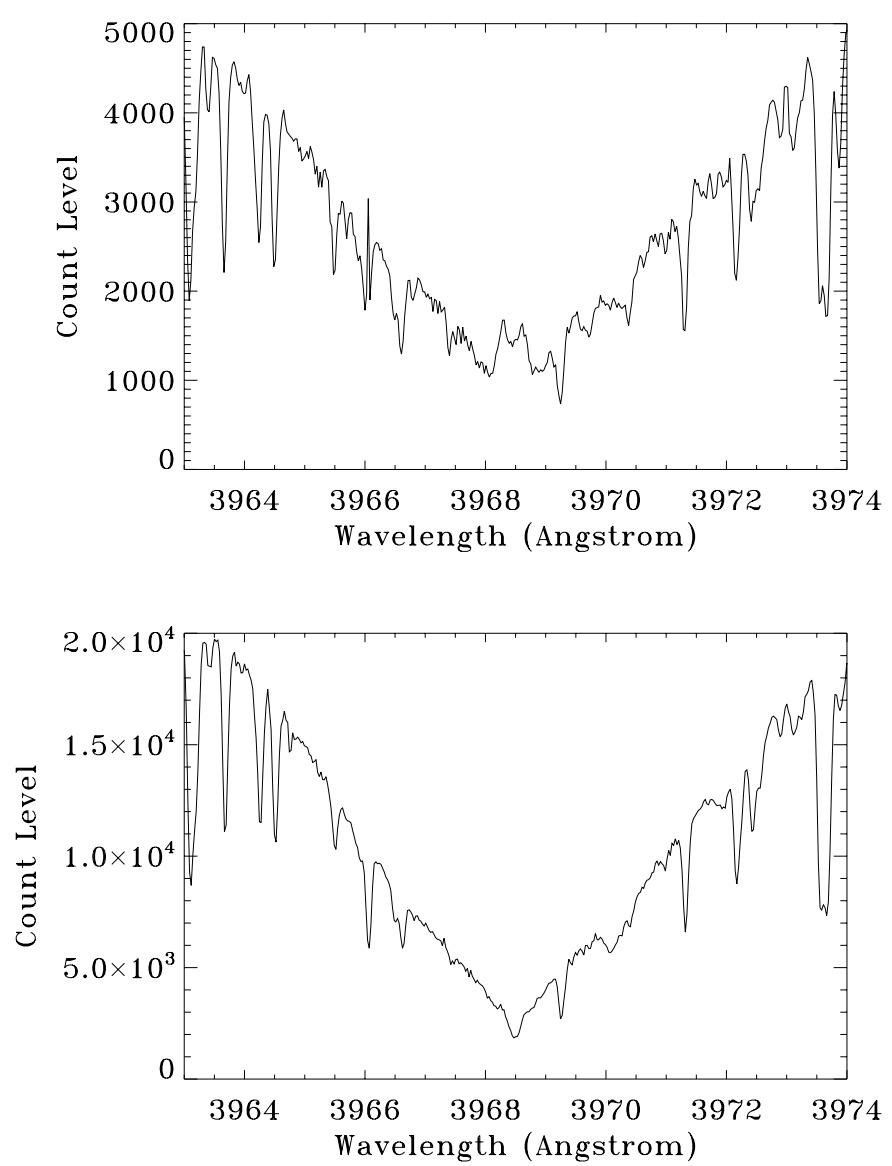

Fig. 2. Comparison between the Ca II H line for HD 81040 (upper panel) and an inactive star of the same temperature (lower panel).

\subsection{Youth and activity indicators}

Montes et al. (2001), on the basis of its galactic kinematics, classify HD 81040 as a young Galactic disc star, with no clear membership to any stellar kinematic group. The star had been selected in that study based on its chromospheric activity levels as measured by Strassmeier et al. (2000) in the Vienna-KPNO search for Doppler-imaging candidate stars. For HD 81040, Strassmeier et al. (2000) measured a value of the chromospheric emission ratio $\log R_{\mathrm{HK}}^{\prime}=-4.52$. All our Keck/HIRES spectra show significant core reversal of the $\mathrm{Ca}$ II $\mathrm{H}$ and $\mathrm{K}$ lines. In the top panel of Fig. 2 we show a region of the HIRES template spectrum centered on the $\mathrm{Ca}$ II $\mathrm{H}$ line. The emission feature is clearly visible. For comparison, in the bottom panel we show the spectrum of an old, inactive star (HIP 105888) with the same temperature from the Sozzetti et al. (2005) sample of metal-poor stars, which has a metallicity of $[\mathrm{Fe} / \mathrm{H}]=-0.72$.

We have measured the Mount Wilson chromospheric activity index S (Duncan et al. 1991) from the Ca II H and $\mathrm{K}$ lines in the Keck/HIRES spectra, and converted it to $R_{\mathrm{HK}}^{\prime}$, following the approach described in Sozzetti et al. (2004). Based on four data-points, the average activity level measured in HD 81040, $\left\langle\log R_{\mathrm{HK}}^{\prime}\right\rangle=-4.48$, agrees very well with that reported by Strassmeier et al. (2000). Based on the Noyes et al. (1984) empirical calibrations, the mean inferred chromospheric age for HD 81040 is then $\langle t\rangle=0.73 \pm 0.1 \mathrm{Gyr}$, and the average stellar

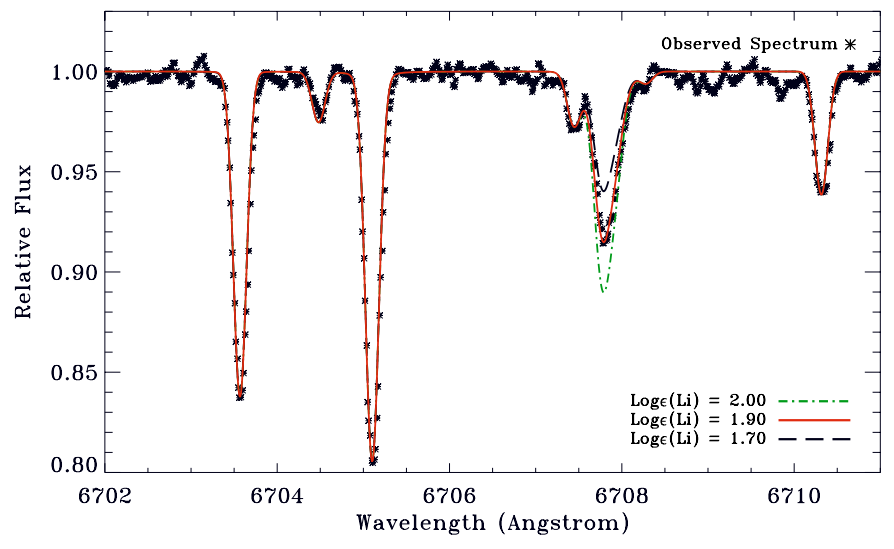

Fig. 3. A portion of the ELODIE co-added spectrum of HD 81040 containing the Li I line at $6707.8 \AA$ (asterisks), compared to three syntheses (lines of different colours and styles). A significant feature is clearly detected.

rotation period $\left\langle P_{\text {rot }}\right\rangle=9.8$ days. On the other hand, the stellar projected rotational velocity appears low. Its measured value, using the mean ELODIE cross-correlation function (CCF) dip width, is $v \sin i=2 \pm 1 \mathrm{~km} \mathrm{~s}^{-1}$, in excellent agreement with the one $\left(2.0 \mathrm{~km} \mathrm{~s}^{-1}\right)$ reported by Nordström et al. (2004). Our estimate of $v \sin i$ is also compatible with the two values of 3.7 and $4.9 \mathrm{~km} \mathrm{~s}^{-1}$ (with typical uncertainties of $2-4 \mathrm{~km} \mathrm{~s}^{-1}$ ) quoted by Strassmeier et al. (2000).

Another important piece of circumstantial evidence in favor of a young age for HD 81040 is the presence of a significant Lithium $(\mathrm{Li})$ feature. In order to derive an estimate of the $\mathrm{Li}$ abundance, we summed all the ELODIE spectra in the region of the $\lambda=6707.8 \AA$ line. We then utilized the resulting co-added ELODIE spectrum to carry out a spectral synthesis of the Li line, using the atmospheric parameters derived from the $\mathrm{Fe}$-line analysis and the same line list of Reddy et al. (2002). In Fig. 3 we show the comparison of the spectrum of HD 81040 with three different models, differing only in the $\mathrm{Li}$ abundance. The best-fit model gives as a result $\log \epsilon(\mathrm{Li})=1.90$. Following Naef et al. (2001b) and Perrier et al. (2003), we also directly measured the equivalent width $\mathrm{EW}_{\lambda}$ of the $\lambda=6707.8 \AA \mathrm{Li}$ I line, and used the Soderblom et al. (1993) curves of growth to obtain a second, independent assessment of the $\mathrm{Li}$ abundance. The resulting value is $\log \epsilon(\mathrm{Li})=1.91 \pm 0.07$, where the error estimate corresponds to $1-\sigma$ changes in $\mathrm{EW}_{\lambda}$ and $T_{\text {eff. }}$. Thus, the two methods we used to determine the $\mathrm{Li}$ abundance in HD 81040 are in very good agreement with each other, and our numbers are also compatible with the value of $\log \epsilon(\mathrm{Li})=2.13 \pm 0.15$ quoted by Strassmeier et al. (2000). Our estimated Li abundance is consistent with the values typical for stars with the same temperature as HD 81040 and slightly older than the Hyades cluster (in good agreement with the chromospheric age estimate), both on an empirical as well as theoretical basis (see for example Sestito \& Randich 2005).

\subsection{Study of radial-velocity jitter}

The excess jitter in the radial-velocity residuals for HD 81040, $\sigma_{v}^{\prime}=\sqrt{\sigma_{\mathrm{O}-\mathrm{C}}^{2}-\left\langle\sigma_{\mathrm{RV}}\right\rangle^{2}} \approx 22 \mathrm{~m} \mathrm{~s}^{-1}$, can be attributed to 

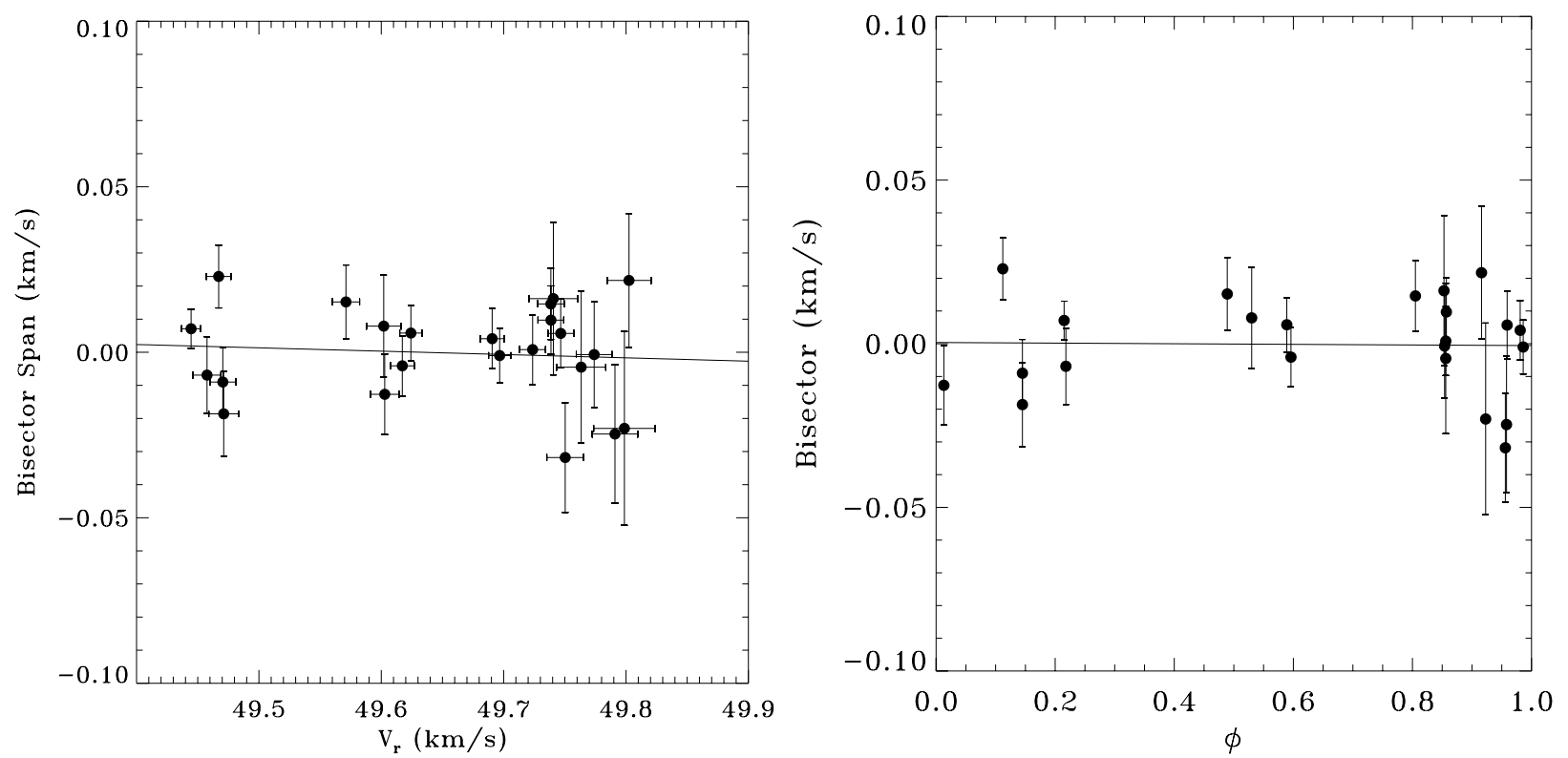

Fig. 4. Left: bisector inverse slope of the ELODIE CCFs (as defined in Queloz et al. 2001) as a function of the radial velocities obtained from the CCFs. A linear fit to the data (solid line) has a statistically insignificant slope of $-0.010( \pm 0.026) V_{r}$. A rank-correlation test gave a probability of no-correlation $p=0.41$. Right: bisector span as a function of orbital phase $\phi$. A linear fit to the data (solid line) has a statistically insignificant slope of $-0.0009( \pm 009) \phi$. A rank-correlation test gave a probability of no-correlation $p=0.58$.

chromospheric activity. It is well known (e.g., Saar et al. 1998; Santos et al. 2000a) that spectral line profile variations induced by surface activity (e.g., spots) can translate into excess radialvelocity jitter. For an early-G dwarf with the level of activity exhibited by HD 81040 , empirical estimates (e.g., Saar et al. 1998; Santos et al. 2000a; Paulson et al. 2002; Wright 2005) indicate a typical value of the activity-induced radial-velocity jitter of $\sigma_{v}^{\prime} \simeq 20 \mathrm{~m} \mathrm{~s}^{-1}$, in good agreement with the observed scatter in the post-fit velocity residuals for HD 81040.

Despite the excess radial-velocity jitter, the orbital motion of HD 81040 is large enough and coherent enough to give a convincing Doppler signature over more than two orbital cycles. The estimated rotation period is about two orders of magnitude shorter than the inferred orbital period, and the radialvelocity semi-amplitude is almost an order of magnitude larger than the presumed activity-induced level of jitter. Unlike the case, for example, of the short-period variable HD 166435 (Queloz et al. 2001), stellar surface activity is very unlikely to be source of the observed radial-velocity curve.

To put this conclusion on firmer grounds, we have carried out an analysis of the line bisectors from the ELODIE CCFs, searching for possible correlations between the bisector span and radial-velocity measurements, orbital phase, and velocity residuals. Rank-correlation tests gave probabilities of no correlation ranging between 0.41 and 0.92 . As an example, in the two panels of Fig. 4 we show the bisector span values plotted against the velocities obtained from the ELODIE CCFs and the orbital phase, respectively. No trend is visible, further supporting the interpretation of true stellar reflex motion induced by an orbiting companion as opposed to radial-velocity variations originating in the stellar atmosphere. Unfortunately, the $R_{\mathrm{HK}}^{\prime}$ measurements are too few to allow a search for periodicities. Finally, Hipparcos lists HD 81040 as photometrically stable, with a scatter of 9 mmag. No significant signal at any frequency was found as a results of a periodogram analysis on the photometric data.

\section{Summary and discussion}

We have presented in this work a combined Keck/HIRES + ELODIE dataset of radial-velocity measurements for the young disc star HD 81040. The measurements reveal the presence of a massive $\left(m_{2} \sin i=6.86 M_{\text {Jup }}\right)$ planetary companion, on an eccentric ( $e=0.53)$, relatively long-period ( $P=1001$ days $)$ orbit. The host star is a $\mathrm{G} 2 / \mathrm{G} 3$ dwarf with a metallicity $[\mathrm{Fe} / \mathrm{H}]=$ -0.16 , very close to the average $([\mathrm{Fe} / \mathrm{H}] \simeq-0.1)$ of the solar neighbourhood (Nordström et al. 2004), but somewhat metaldeficient with respect to the average $([\mathrm{Fe} / \mathrm{H}] \simeq 0.14)$ of the metallicity distribution of planet-hosting stars (e.g., Fischer \& Valenti 2005). Both the chromospheric activity level (measured using the $\mathrm{Ca}$ II $\mathrm{H}$ and $\mathrm{K}$ lines in the HIRES spectra) as well as the Lithium abundance (estimated using the coadded ELODIE spectrum) speak in favor of a young age for HD $81040(\approx 0.8 \mathrm{Gyr})$, in good agreement with previous studies of this object (Strassmeier et al. 2000; Montes et al. 2001).

The excess scatter $\left(\sim 22 \mathrm{~m} \mathrm{~s}^{-1}\right)$ in the velocity residuals from the best-fit orbit compared to the nominal internal errors of the HIRES and ELODIE measurements can be attributed to activity on the surface of HD 81040. Radial-velocity surveys of nearby F-G-K dwarfs tend to avoid chromospherically active stars, because velocity jitter caused by surface activity not only degrades the velocity precision, but can even mimic the signal produced by an orbiting companion (e.g., Queloz et al. 2001). However, HD 81040 is not the first young star found to harbor a planetary-mass companion despite its significant activity levels. We summarize in Table 4 the properties of active 
Table 4. Main properties of active planet-hosting stars and their orbiting planets. Only objects with a chromospheric age estimate $t<1.5 \mathrm{Gyr}$ are included (corresponding to $\log R_{\mathrm{HK}}^{\prime}>-4.65$ ). Columns 1 through 12 report: star name, spectral type, metallicity, projected rotational velocity, chromospheric emission ratio, lithium abundance or upper limit (where applicable), estimated rotation period, chromospheric age, radial velocity jitter estimate, orbital periods of the companions, minimum masses, and radial-velocity semi-amplitude. Unless otherwise noted, all parameter values are taken from the literature sources listed in Col. 13. The star HD 166435 does not have a planet, but is listed for comparison.

\begin{tabular}{|c|c|c|c|c|c|c|c|c|c|c|c|c|}
\hline Star & $\begin{array}{c}\text { Sp. } \\
\text { type }\end{array}$ & {$[\mathrm{Fe} / \mathrm{H}]$} & $\begin{array}{c}v \sin i \\
\left(\mathrm{~m} \mathrm{~s}^{-1}\right)\end{array}$ & $\log R_{\mathrm{HK}}^{\prime}$ & $\log \epsilon(\mathrm{Li})^{a}$ & $\begin{array}{c}P_{\text {rot }}{ }^{b} \\
\text { (days) }\end{array}$ & $\begin{array}{l}\text { Age }^{c} \\
\text { (Gyr) }\end{array}$ & $\begin{array}{c}\sigma_{v}^{\prime d} \\
\left(\mathrm{~m} \mathrm{~s}^{-1}\right)\end{array}$ & $\begin{array}{c}P \\
\text { (days) }\end{array}$ & $\begin{array}{c}m_{2} \sin i \\
\left(M_{\text {Jup }}\right)\end{array}$ & $\begin{array}{c}K_{1} \\
\left(\mathrm{~m} \mathrm{~s}^{-1}\right)\end{array}$ & Refs. $^{e}$ \\
\hline HD 81040 & G2/G3 & -0.16 & 2.0 & -4.48 & 1.90 & 10 & 0.7 & $7 / 19$ & 1001 & 6.86 & 168 & (1) \\
\hline HD 1237 & G6V & +0.10 & 5.5 & $-4.44^{f}$ & 2.24 & 10 & 0.6 & $21 / 21$ & 133 & 3.32 & 164 & (2) \\
\hline HD 17051 & G0V & +0.03 & 6.1 & -4.65 & 2.63 & 12 & 1.5 & $13 / 14$ & 320 & 2.26 & 67 & (3) \\
\hline HD 22049 & $\mathrm{~K} 2 \mathrm{~V}$ & -0.10 & $2.5^{g}$ & $-4.47^{h}$ & $<0.3$ & 10 & 0.7 & $9 / 9$ & 2502 & 0.86 & 18 & (4) \\
\hline HD 40979 & F8 & +0.19 & 7.4 & -4.63 & 2.79 & 12 & 1.0 & $19 / 15$ & 263 & 3.28 & 100 & (5) \\
\hline HD 73256 & G8/K0 & +0.29 & 3.2 & -4.49 & $\ldots$ & 12 & 0.8 & $13 / 19$ & 250 & 1.87 & 265 & (6) \\
\hline HD 121504 & G2V & +0.16 & 2.6 & -4.57 & 2.66 & 9 & 1.1 & $7 / 16$ & 63 & 1.22 & 45 & (7) \\
\hline \multirow[t]{2}{*}{ HD 128311} & K0 & +0.08 & 5.7 & -4.44 & $<-0.4$ & 10 & 0.5 & $11 / 9$ & 458 & 2.18 & 67 & (8) \\
\hline & & & & & & & & & 928 & 3.21 & 76 & \\
\hline HD 130322 & K0 & -0.02 & 1.9 & -4.39 & $<0.2$ & 8 & 0.3 & $7 / 9$ & 10 & 1.02 & 115 & (9) \\
\hline HD 141937 & G2/G3V & +0.01 & 2.1 & -4.65 & 2.48 & 13 & 1.5 & $6 / 14$ & 653 & 9.70 & 247 & (10) \\
\hline HD 142415 & G1V & +0.21 & 3.3 & -4.55 & & 10 & 1.1 & $8 / 16$ & 386 & 1.62 & 51 & (7) \\
\hline HD 147513 & $\mathrm{G} 3 / 5 \mathrm{~V}$ & +0.06 & 1.5 & -4.38 & 2.05 & 5 & 0.3 & $4 / 23$ & 528 & 1.21 & 31 & (7) \\
\hline HD 192263 & $\mathrm{~K} 2 \mathrm{~V}$ & -0.14 & 1.8 & -4.39 & $<-0.3$ & $9^{i}$ & 0.3 & $6 / 9$ & 24 & 0.72 & 62 & (11) \\
\hline HD 196050 & G3V & +0.22 & 3.1 & $-4.65^{j}$ & 2.15 & 16 & 1.5 & $9 / 14$ & 1321 & 3.02 & 49 & (7) \\
\hline HD 166435 & G0 & -0.07 & 7.6 & -4.26 & $<1.7$ & $4^{k}$ & 0.2 & $33 / 28$ & 4 & 0.60 & 83 & (12) \\
\hline
\end{tabular}

a All abundances and upper limits are taken from Israelian et al. (2004), except for HD 81040 (this work), HD 40979 (Fischer et al. 2002 ) and HD 166435 (Queloz et al. 2001).

${ }^{b}$ From the Noyes et al. (1984) calibrations.

$c$ From the Noyes et al. (1984) calibrations.

${ }^{d}$ The two values are derived using the Saar et al. (1998) and the Santos et al. (2000a) calibrations, respectively.

e (1): this work; (2): Naef et al. (2001b); (3) Kürster et al. (2000); (4): Hatzes et al. (2000); (5): Fischer et al. (2002); (6): Udry et al. (2003); (7): Mayor et al. (2004); (8): Vogt et al. (2005); (9): Udry et al. (2000); (10): Udry et al. (2002); (11): Santos et al. (2000b); (12): Queloz et al. (2001).

${ }^{f}$ From Henry et al. (1996). Naef et al. (2001b) report a substantially higher value $\log R_{\mathrm{HK}}^{\prime}=-4.27$ (but with large scatter), corresponding to $P_{\text {rot }}=4$ days and $t=0.02$ Gyr.

${ }^{g}$ From Tokovinin (1992). Uncertainties on the $v \sin i$ value for HD 22049 are rather large.

${ }^{h}$ From Henry et al. (1996).

${ }^{i}$ A photometric period of 24 days, matching the orbital period, was obtained by Henry et al. (2002). However, Santos et al. (2003) have shown the planet hypothesis still holds.

${ }^{j}$ Henry et al. (1996) report a much lower value $\log R_{\mathrm{HK}}^{\prime}=-5.04$.

${ }^{k}$ The photometric period matches the orbital and chromospheric period estimates. Queloz et al. (2001) have shown the observed signal is due to activity-related processes.

$\left(\log R_{\mathrm{HK}}^{\prime}>-4.65\right.$, corresponding to a chromospheric age estimate $t<1.5 \mathrm{Gyr}$ ) stars with known detected planets, and the main characteristics of the latter. At least two important conclusions can be drawn from Table 4.

First, the two main youth indicators $\left(\log R_{\mathrm{HK}}^{\prime}\right.$ and, when measurable, $\log \epsilon(\mathrm{Li}))$, and to a lesser extent $v \sin i$, are in fair agreement with each other. In the upper panel of Fig. 5 we plot as a function of age the chromospheric flux in the Ca II $\mathrm{K}$ line cores $F_{\mathrm{K}}^{\prime}$ (expressed in erg $\mathrm{cm}^{-2} \mathrm{~s}^{-1}$ ) for the planet hosts in Table 4 compared to the results for open clusters and empirical curves from Pace \& Pasquini (2004), and references therein. The second panel shows the corresponding comparison for $v \sin i$ versus age. The agreement is good. A similar result holds for the comparison between average $\mathrm{Li}$ abundances in open clusters from Sestito \& Randich (2005) and the average $\log \epsilon(\mathrm{Li})$ for the ensemble of active stars with planets (lower panel of Fig. 5). The average age inferred from the chromospheric activity values for the stars in Table 4 is $\sim 0.8 \mathrm{Gyr}$, slightly older than the Hyades cluster. The average values of $F_{\mathrm{K}}^{\prime}, v \sin i$, and $\log \epsilon(\mathrm{Li})$ are consistent with such interpretation.

Second, to appreciate how severe a danger (moderate) youth and activity can pose for radial-velocity planet surveys, it is useful to compare the values of radial-velocity semiamplitude and orbital period of the companions with the expected levels of jitter (carrying typical uncertainties of order $30 \%-50 \%$ ) and estimated stellar rotation periods reported in Table 4 . When both $K_{1} \gg \sigma_{v}^{\prime}$ and $P \gg P_{\text {rot }}$, conditions realized in the majority of the cases presented in Table 4 , there can be little doubt that the orbital motion is real. If $K_{1}>\sigma_{v}^{\prime}$, but $P$ is similar to $P_{\text {rot }}$ (as is the case for HD 130322 and HD 192263), simultaneous radial-velocity, photometry, $\mathrm{Ca}$ II, and line 

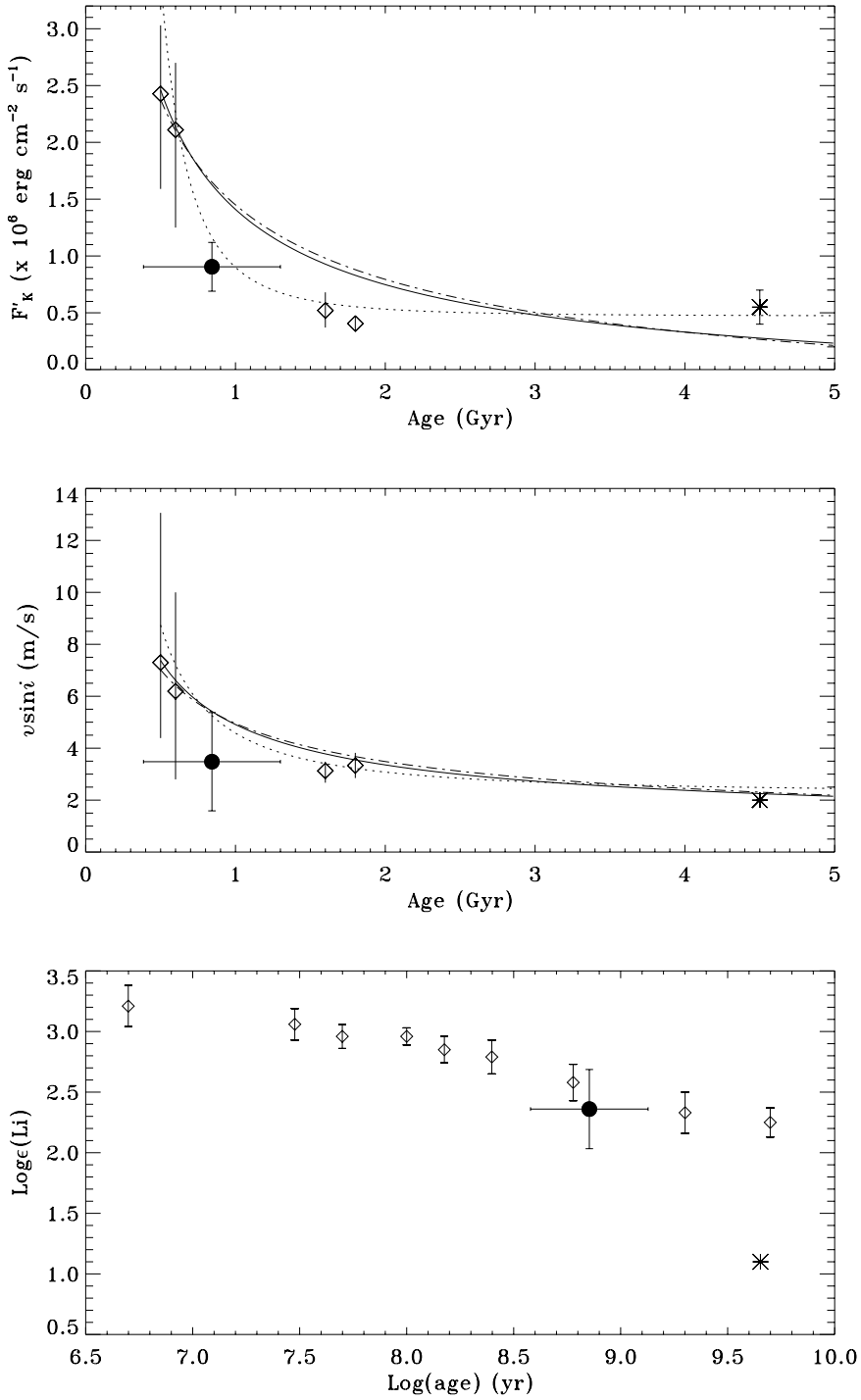

Fig. 5. Top: average chromospheric flux $F_{\mathrm{K}}^{\prime}$ (corrected for the photospheric-flux contribution) as a function of age for four intermediate-age clusters (diamonds), the sun (asterisk), and active stars with planets (filled circle). For clusters, error bars represent peakto-peak spreads. For stars with planets, they correspond to the spread around the mean value. Lines of different shapes represent theoretical power-law fits to the data with different exponents (Pace \& Pasquini 2004). Center: the same, but in the $v \sin i$-age plane. Bottom: average lithium abundance $\log \epsilon(\mathrm{Li})$ as a function of age for open clusters (diamonds) and active planet hosts (filled circle). For the cluster averages, only G-type stars are considered (Sestito \& Randich 2005). For stars with planets, only actual $\log \epsilon(\mathrm{Li})$ estimates (no upper limits) have been used. All error bars correspond to the dispersion around the mean value.

bisector measurements must be carried out in order to ascertain that the planet is real and the observed signal is not intrinsic to the star (as in the case of HD 166435, also reported in Table 4 for comparison). If instead $P \gg P_{\text {rot }}$, but $K_{1}$ is comparable to $\sigma_{v}^{\prime}$, the situation becomes more complex, as long-term activity cycles may reproduce very low-amplitude pseudo-periodic signals, which vary from season to season (e.g., Cumming et al. 1999).
For example, based on the absence of a periodicity in the $R_{\mathrm{HK}}^{\prime}$ time-series similar to that of the orbital signal, Hatzes et al. (2000) claimed HD 22049 actually harbors a planet, but this evidence had not been universally accepted in the past as a strong argument in favor of the planet hypothesis (e.g., Butler et al. 2003). In this specific case, the planet existence has been confirmed (Benedict 2005, private communication) by the recently completed HST astrometry campaign (Benedict et al. 2003, 2004) on this star.

Based on all the available evidence, the existence of a planetary-mass companion orbiting HD 81040 appears to be the best explanation for the observed radial-velocity variations. The inferred minimum mass for the object places it in the high-mass tail of the extrasolar planet distribution, i.e. the $18 \%$ or so of detected planets around nearby F-G-K stars with $m_{2} \sin i \gtrsim 5 M_{\text {Jup }}$. This region of the planet mass distribution is populated by objects with minimum masses close to and even larger than the widely used but arbitrary dividing line between planets and brown dwarfs of $13 M_{\text {Jup }}$ (e.g., Oppenheimer et al. 2000). Some of the objects with minimum masses above the $13 M_{\text {Jup }}$ deuterium-burning limit can make strong claims for being called planets, because they occur in systems containing another companion with lower-mass. Good examples are HD 168443 (two companions with $m \sin i=7.7 M_{\text {Jup }}$ and $m \sin i=17.2 M_{\text {Jup }}$; Marcy et al. 2001; Udry et al. 2002) and HD 202206 (two companions with $m \sin i=2.4 M_{\text {Jup }}$ and $m \sin i=17.4 M_{\mathrm{Jup}}$; Correia et al. 2005).

The observed paucity of objects in the gap between the high-mass tail for the planets on the one hand and the lowmass tail for stellar companions with spectroscopic orbits on the other hand is often called the "brown dwarf desert" (e.g., Halbwachs et al. 2000). Regardless of whether they are called giant planets or brown dwarfs, the most massive objects at the lower edge of the desert are important because they may help discriminate between the core accretion and disk instability models for planet formation. For example, recent models of giant planet formation that start with the accretion of a rocky core (e.g., Lissauer 1993; Pollack et al. 1996) can qualitatively reproduce the observed mass distribution of extrasolar planets (Alibert et al. 2005; Ida $\&$ Lin 2004a, 2005) from $M \lesssim 5 M_{\text {Jup }}$ down to the Neptune-mass (and lower) regime of some recently discovered objects (McArthur et al. 2004; Butler et al. 2004; Santos et al. 2004; Rivera et al. 2005). Planets more massive than $M \sim 5 M_{\mathrm{Jup}}$ are more difficult to form by core accretion. In contrast, the alternative disk-instability model (e.g., Boss 2001, 2005; Mayer et al. 2004) predicts that objects formed by this mechanism can populate the high-mass tail ( $\left.M \gtrsim 5 M_{\text {Jup }}\right)$ of the planet mass distribution (Rice et al. 2003; Rafikov 2005). Improved statistics on the frequency of massive planets and their properties, together with refined theoretical studies of the formation of massive planets (and of their relative frequency with respect to lower-mass planets) may help us understand the relative roles of these two formation mechanisms.

Finally, given the projected separation of 62 mas, and given the relatively large mass and presumably rather young age of the companion, HD 81040 could be an interesting candidate for observations with future, direct near- and far-infrared imaging surveys of wide-separation giant planets (e.g., Burrows 2005, 
and references therein). Also, the magnitude of the inferred astrometric signature might also make this system an attractive target for high-precision ground-based as well as space-borne astrometric surveys (e.g., Sozzetti 2005, and references therein) which will come online in the near future.

Acknowledgements. A.S. gratefully acknowledges financial support through the Keck PI Data Analysis Fund (JPL 1262605). S.Z. is grateful for partial support from the Jacob and Riva Damm Foundation. G.T. acknowledges partial support for this work from NASA Origins grant NNG04LG89G. We wish to thank D. Yong for valuable discussion. The referee, William Cochran, provided very helpful comments and suggestions. We are grateful to the Observatoire de HauteProvence for the generous time allocation. This research has made use of NASA's Astrophysics Data System Abstract Service and of the SIMBAD database, operated at CDS, Strasbourg, France.

\section{References}

Alibert, Y., Mordasini, C., Benz, W., \& Winisdoerffer, C. 2005, A\&A, 434,343

Allende Prieto, C., \& Lambert, D. L. 1999, A\&A, 352, 555

Baliunas, S. L., Donahue, R. A., Soon, W. H., et al. 1995, ApJ, 438, 269

Baranne, A., Queloz, D., Mayor, M., et al. 1996, A\&AS, 119, 373

Benedict, G. F., McArthur, B. E., the Space Telescope Astrometry Team and the "Are These Planets or Not?" Team 2003, BAAS, 35, \#67.05

Benedict, G. F., McArthur, B. E., the Space Telescope Astrometry Team and the "Are These Planets or Not?" Team 2004, BAAS, $36, \# 42.02$

Boss, A. P. 2001, Nature, 409, 462

Boss, A. P. 2005, ApJ, 629, 535

Burrows, A. 2005, Nature, 433, 261

Butler, R. P., Marcy, G. W., Williams, E., et al. 1996, PASP, 108, 500

Butler, R. P., Marcy, G. W., Vogt, S. S., et al. 2003, ApJ, 582, 455

Butler, R. P., Vogt, S. S., Marcy, G. W., et al. 2004, ApJ, 617, 580

Cochran, W. D., Hatzes, A. P., \& Paulson, D. B. 2002, AJ, 124, 565

Correia, A. C. M., Udry, S., Mayor, M., et al. 2005, A\&A, 440, 751

Cox, A. N. 2000, Allen's Astrophysical Quantities, 4th, edn. (New York: Springer-Verlag)

Cumming, A., Marcy, G. W., \& Butler, R. P. 1999, ApJ, 526, 890

Duncan, D. K., Vaughan, A. H., Wilson, O. C., et al. 1991, ApJS, 76, 383

Fischer, D. A., \& Valenti, J. 2005, ApJ, 622, 1102

Fischer, D. A., Marcy, G. W., Butler, R. P., et al. 2002, ApJ, 586, 1394

Halbwachs, J. L., Arenou, F., Mayor, M., Udry, S., \& Queloz, D. 2000, A\&A, 355, 581

Hatzes, A. P., Cochran, W. D., McArthur, B., et al. 2000, ApJ, 544, L145

Henry, T. J., Soderblom, D. R., Donahue, R. A., \& Baliunas, S. L. 1996, AJ, 111, 439

Henry, G. W., Donahue, R. A., \& Baliunas, S. L. 2002, ApJ, 577, L111

Kürster, M., Endl, M., Els, S., et al. 2000, A\&A, 353, L33

Ida, S., \& Lin, D. N. C. 2004, ApJ, 604, 388

Ida, S., \& Lin, D. N. C. 2005, ApJ, 626, 1045

Israelian, G., Santos, N. C., Mayor, M., \& Rebolo, R. 2004, A\&A, 414,601

Latham, D. W. 2000, in Disks, Planetesimals, and Planets, ed. F. Garzòn, C. Eiroa, D. de Winter, \& T. J. Mahoney, ASP Conf. Ser., 219, 596

Lissauer, J. J. 1993, ARA\&A, 31, 129

Marcy, G. W., \& Butler, R. P. 1992, PASP, 104, 270
Marcy, G. W., Butler, R. P., Vogt, S. S., et al. 2001, ApJ, 555, 418

Marcy, G. W., Butler, R. P., Vogt, S. S., et al. 2005a, ApJ, 619, 570

Marcy, G. W., Butler, R. P., Fischer, D. A., et al. 2005b, Progress of Theoretical Physics Supplement, 158, 24

Mayer, L., Quinn, T., Wadsley, J., \& Stadel, J. 2004, ApJ, 609, 1045

Mayor, M., \& Queloz, D. 1995, Nature, 378, 355

Mayor, M., Udry, S., Naef, D., et al. 2004, A\&A, 415, 391

Mazeh, T., Naef, D., Torres, G., et al. 2000, ApJ, 532, L55

McArthur, B. E., Endl, M., Cochran, W. D., et al. 2004, ApJ, 614, L81

Mihalas, D., \& Binney, J. 1981, Galactic astronomy: Structure and kinematics, 2nd Ed. (San Francisco, CA: W. H. Freeman and Co.)

Montes, D., López-Santiago, J., Gálvez, M. C., et al. 2001, MNRAS, 328,45

Naef, D., Latham, D. W., Mayor, M., et al. 2001a, A\&A, 375, L27

Naef, D., Mayor, M., Pepe, F., et al. 2001b, A\&A, 375, 205

Nordström, B., Mayor, M., Andersen, J., et al. 2004, A\&A, 418, 989

Noyes, R. W., Hartmann, L. W., Baliunas, S. L., Duncan, D. K., \& Vaughan, A. H. 1984, ApJ, 279, 763

Oppenheimer, B. R., Kulkarni, S. R., \& Stauffer, J. R. 2000, in Protostars and Planets IV, ed. V. Mannings, A. P. Boss, \& S. S. Russell (Tucson: University of Arizona Press), 1313

Pace, G., \& Pasquini, L. 2004, A\&A, 426, 1021

Paulson, D. B., Saar, S. H. Cochran, W. D., \& Hatzes, A. P. 2002, AJ, 124,572

Paulson, D. B., Sneden, C., \& Cochran, W. D. 2003, AJ, 125, 3185

Perrier, C., Sivan, J.-P., Naef, D., et al. 2003, A\&A, 410, 1039

Pollack, J. B., Hubickyj, O., Bodenheimer, P., et al. 1996, Icarus, 124, 62

Queloz, D., Henry, G. W., Sivan, J. P., et al. 2001, A\&A, 379, 279

Rafikov, R. R. 2005, ApJ, 621, L69

Reddy, B. E., Lambert, D. L., Laws, C., Gonzalez, G., \& Covey, K. 2002, MNRAS, 335, 1005

Rice, W. K. M., Armitage, P. J., Bonnell, I. A., et al. 2003, MNRAS, 346, L36

Rivera, E., Lissauer, J. J., Butler, R. P., et al. 2005, ApJ, 634, 625

Saar, S. H., Butler, R. P., \& Marcy, G. W. 1998, ApJ, 498, L153

Santos, N. C., Mayor, M., Naef, D., et al. 2000a, A\&A, 361, 265

Santos, N. C., Mayor, M., Naef, D., et al. 2000b, A\&A, 356, 599

Santos, N. C., Bouchy, F., Mayor, M., et al. 2004, A\&A, 426, L19

Sestito, P., \& Randich, S. 2005, A\&A, 442, 615

Soderblom, D. R., Jones, B. F., Balachandran, S., et al. 1993, AJ, 106, 1059

Sozzetti, A. 2005, PASP, 117, 1021

Sozzetti, A., Yong, D., Torres, G., et al. 2004, ApJ, 616, L167

Sozzetti, A., Latham, D. W., Torres, G., et al. 2005, in Gaia: The Three-Dimensional Universe, ESA-SP, 576, 309

Sozzetti, A., Yong, D., Carney, B. W., et al. 2006, AJ, in press [arXiv:astro-ph/0512510]

Strassmeier, K., Washuettl, A., Granzer, Th., Scheck, M., \& Weber, M. 2000, A\&A, 142, 275

Tokovinin, A. A. 1992, A\&A, 256, 121

Udry, S., Mayor, M., Naef, D., et al. 2000, A\&A, 356, 590

Udry, S., Mayor, M., Naef, D., et al. 2002, A\&A, 390, 267

Udry, S., Mayor, M., Clausen, J. V., et al. 2003, A\&A, 407, 679

Valenti, J. A., Butler, R. P., \& Marcy, G. W. 1995, PASP, 107, 966

Vogt, S. S., Allen, S. L., Bigelow, B. C., et al. 1994, in Instrumentation in Astronomy VIII, ed. D. L. Crawford, \& E. R. Craine, Proc. SPIE, 2198, 362

Vogt, S. S., Butler, R. P., Marcy, G. W., et al. 2005, ApJ, 632, 638

Wright, J. T. 2005, PASP, 117, 657

Yi, S., Kim, Y. -C., \& Demarque, P. 2003, ApJS, 144, 259

Zucker, S., \& Mazeh, T. 2001, ApJ, 562, 549

Zucker, S., Naef, D., Latham, D. W., et al. 2002, ApJ, 568, 363 